\title{
Priority Incorporated Zone Based Distributed Clustering Algorithm for Heterogeneous Wireless Sensor Network
}

Hamid Ali Abed AL-Asadi *,1,2, Abdulhadi Alhassani ${ }^{1}$, Nor Azura Ahmed Hambali ${ }^{3}$, Mustafa Abdulazeez AlSibahee ${ }^{1,4}$, Saif Ali Alwazzan $^{1}$, Ali Mohammed Jasim ${ }^{1}$

${ }^{1}$ Communications Engineering Department, Iraq University College, Basra, 61004, Iraq.

${ }^{2}$ Computer Science Department, Basra University, Basra, 61004, Iraq

${ }^{3}$ Semiconductor Photonics \& Integrated Lightwave Systems (SPIILS), School of Microelectronic Engineering, Universiti Malaysia Perlis, Pauh Putra Main Campus, 02600 Arau, Perlis, Malaysia

${ }^{4}$ Shenzhen Institute of Huazhong University of Science and Technology, Shenzhen, China

\begin{tabular}{l} 
A R T I C L E I N F O \\
\hline Article history: \\
Received: 29 July, 2019 \\
Accepted: 21 September, 2019 \\
Online: 15 October, 2019
\end{tabular}

Keywords:

Heterogeneous wireless sensor network

Priority

Zone based clustering

Distributed clustering

Supporting cluster head

Clustering

Sensor nodes

\begin{abstract}
A B S T R A C T
Wireless sensor networks (WSNs) are considered to be the currently flourishing scientific domain, thereby found to be applicable in numerous industrial and domestic applications. As per the mathematical results in Pulse-coupled oscillator (PCO), it has been predicted that, numerous iterations are needed for convergence, leading to increased power consumption. Biologically inspired solutions are greatly applicable for recovering coverage issues and efficient routing processes. In Hybrid energy efficient distributed clustering (HEED), to find a node with lowest communication cost, large number of iterations is needed, thereby leading to larger time duration for finding such node, and this is considered as the prevalent drawback, resulting in significant power consumption. In Optical low energy adaptive clustering hierarchy $(O-L E A C H)$, the cluster head selection is based on randomness, resulting in easy cluster failure. Hence, for cases, where power minimization and higher network lifetime is to be achieved to a larger extent, the existing strategies shall not be applicable, due to few restrictions. In this paper, a priority incorporated zone based distributed clustering algorithm, the Better Integrated and Optimized Low Energy Adaptive Clustering Hierarchy (BIO-LEACH) has been proposed for heterogeneous WSN. The methodology of this distributed clustering algorithm possesses three distinct features. First, the given clustering area will be divided in to different clusters and each cluster will be assigned with priority. The cluster which is highly sensitive and which needs frequent data recording will be given highest priority. The clusters in which the priority is assigned, takes multiple sensing and communication even in one cycle. But, the clusters where priority is not assigned, only one sensing and data transmission will be allowed for one cycle. Second, the clusters possessing priorities will have one cluster head (CH) and two supporting cluster head (SCH), but the clusters that do not have priorities will have only one cluster head. Third, the clusters possessing priorities will be possessing cluster nodes more than that of the clusters without priorities, so as to avoid cluster failure. Simulation results have been done to evaluate the performance of the proposed algorithm in terms of number of cluster head selection, amount of energy consumed and number packets received.
\end{abstract}

"Corresponding Author Hamid A. AL-Asadi, Head of Communications Engineering Department, Iraq University College, Basra, 61004, Iraq. Computer Science Department, Basra University, Basra, 61004, Iraq. Email: hamid.alasadi@iuc.edu.iq, hamid.abed@uobasrah.edu.iq 


\section{Introduction}

In a typical wireless sensor networks (WSN), there will be hundreds to thousand number of sensor nodes that will be capable of functioning in unattended environments [1]. The sensor nodes in such networks collect information from the predestined environment and communicate with each other in a cooperative manner. These wireless sensor networks form the basic functioning module of Internet of Things (IoT) [2], thereby sensed data can be transmitted over the IoT, for numerous real-world applications like healthcare and military. These sensor networks therefore form the basic research focus in the present era, and also when these sensor nodes function as actuators, they could be intelligent to control a particular situation based on the sensed values or reading. These actuator applications are quite commonly applicable in military applications, earthquake detection and alerting, environmental monitoring applications, etc. Considering a single sensor node, it possesses a sensing unit, microcontroller unit, storage unit, radio communication unit and an antenna [3-5]. But, these sensor nodes are tiny in size, thereby power becomes the main area to be concentrated. Unlike other networks, sensor networks are mostly preferred to function in unattended environments where even human live is merely possible, thereby the power consumption of the batteries have to be clearly controlled. Therefore, bringing out an energy efficient strategy to minimize the power consumption of the sensor nodes has become the main view of recent researchers. One such method to attain these functionalities is by effectively forming clusters.

These sensor networks can be employed to measure single/multiple parameters using the same network, and hence heterogeneous WSN comes in to picture. The main difference between homogenous and heterogeneous wireless sensor network, in one point of view is that, the former uses identical sensor throughout the sensor field, but the latter uses different types of sensors within the sensor field.

Therefore, in a heterogeneous WSN, the main target will be towards reducing the power consumption of the sensor nodes and prolonging the overall lifetime of the network. Heterogeneous WSN possesses a group of sensor nodes that are different in functionalities, thereby deploying them, and topology management is also a serious concern [6].

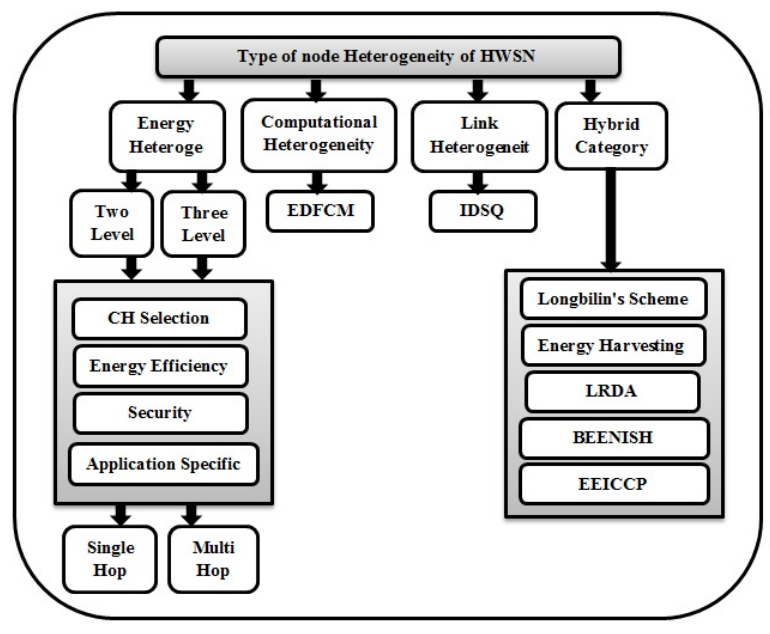

Figure 1: Taxonomy of heterogeneous schemes.
Fig.1 defines the taxonomy of heterogeneous protocols for WSNs. Heterogeneity of a sensor node may be based on energy, computational and link heterogeneities. Energy heterogeneity specifies the replacement capabilities of batteries in a sensor node, as the energy is the main constraint when compared to computational complexities and link failures in a heterogeneous WSN. Computational heterogeneity specifies that the particular sensor node possesses higher features like speed, storage, battery, etc., when compared to ordinary sensor nodes that too directly depends on energy heterogeneity. Processing highly complex data along with increased storage capabilities, forms the specific feature of a heterogeneous wireless sensor network. The communication distance between two sensor nodes is mainly considered as the parameter for link heterogeneity. When we go for long distance communication, communication bandwidth also forms a major concern in a heterogeneous WSN.

This paper has been structured as follows. An introduction to various concepts in a wireless sensor network, mainly focussing on heterogeneity, has been discussed in Section 1. The literature survey of several algorithms towards the closer vicinity of this research work is discussed in Section 2. The features of the proposed BIO-LEACH algorithm, and how it differs from the traditional algorithms has been clearly discussed in Section 3 . Simulation results along with necessary discussions have been elaborated in Section 4. Finally, Section 5 concludes the paper.

\section{Literature Review}

Few related researches work carried out by researchers in the closer vicinity of our research work is discussed here, that includes, Pulse-coupled oscillator (PCO), Biologically inspired solutions, Hybrid energy efficient distributed clustering (HEED) and Optical low energy adaptive clustering hierarchy $(\mathrm{O}-\mathrm{LEACH})$.

Pulse-Coupled Oscillator (PCO) Method: Based on PCO, the first proposal started for attaining synchronization in a heterogeneous WSN. But, prior to that numerous research works have been carried out with theoretical and mathematical models, but they did not concentrate on the hardware part. This hardware oriented PCO strategy mainly concentrated on the physical layer parameters and employed point to point communication topology. But, subsequently many approaches have been proposed based on PCO for ultra wideband (UWB) communication applications [7]. This PCO falls under the category of energy efficient synchronization protocols for heterogeneous WSN. As per the mathematical results, it has been predicted that numerous iterations are needed for convergence. The mapping is done in continuous manner so as to attain convergence in these sensor networks. Even though, higher efforts were taken towards convergence and synchronization, these strategies will not guarantee effective convergence.

Biologically Inspired Solutions: These biologically inspired solutions are typically designed so as to imitate the basic functionalities of naturally inspired metaheuristic. Apart from attaining energy efficiency and prolonging network lifetime, these biologically inspired solutions offer numerous interesting benefits in WSN [8-10]. These strategies are normally considered as an effective tool that are generally simpler, highly scalable and offers distributed approaches for heterogeneous WSNs. These strategies are normally considered as the replica of optimizing real time 


\section{H.A.A AL-Asadi et al. / Advances in Science, Technology and Engineering Systems Journal Vol. 4, No. 5, 306-313 (2019)}

problems. Considering few examples, the ant colony optimization strategy serves as an effective tool for multipath clustering and routing, particle swarm optimization is considered as an effective approach for generating optimal number of clusters, the genetic algorithm is mainly employed to recover the coverage issues, and artificial bee colony algorithm mainly results in formation of efficient clustering and routing processes. These approaches are found to be applicable for controlling automated smart lighting systems and also in photovoltaics [11-14].

Hybrid Energy Efficient Distributed Clustering (HEED): This particular approach is mainly formulated so as to attain effective clusters in a wireless sensor network [15]. HEED algorithm mainly depends upon residual energy and communication cost for forming effective clusters. In case of Optical Low Energy Adaptive Clustering Hierarchy (O-LEACH), the cluster head is selected in random manner, but HEED was proposed for eradication of random cluster head $(\mathrm{CH})$ selection. Initialization, repetition and finalization phases are the three different phases carried out while forming clusters in WSN based on HEED. The initial probability of a node to become $\mathrm{CH}$ for the current round is decided in the initialization phase of HEED. The node with lowest communication cost will be elected as $\mathrm{CH}$ in the repetition phase of HEED. And, in finalization phase, the election of cluster head will however be properly settled. In order to find a node with lowest communication cost, numerous iterations are needed, thereby leads to larger time duration for finding such node and this is considered as the prevalent drawback in HEED.

Optical Low Energy Adaptive Clustering Hierarchy (OLEACH): This strategy was proposed to connect two separate and longer distanced sensor networks using an optical and distributed fiber link, and every other clustering process is followed as per the traditional LEACH algorithm. These two wireless sensor nodes are considered to possess huge number of wireless sensor nodes, and based on the nature of required applications, it could be assigned that these sensors shall communicate or shall not communicate with other sensor nodes. This optical fiber serves as the connectivity between two separate sensor fields, therefore at the edge of the network, base station (BS) will be positioned. The $\mathrm{CH}$ performs data aggregation process so as to further reduce the quantity of data that needs to be communicated to the base station [16-17]. As per the traditional LEACH concept, the cluster head selection is carried out in a random manner, and this is mainly considered as the common drawback during the cluster formation in O-LEACH algorithm also. The aggregated data is forwarded from the cluster head to the base station either by direct forward or hopping through other cluster heads.

\subsection{Problem Statement}

Considering heterogeneous network, as per the mathematical results in $\mathrm{PCO}$, it has been predicted that, numerous iterations are needed for convergence, leading to increased power consumption. Hence, for cases, where power minimization and higher network lifetime is to be achieved, this strategy shall not be applicable. Biologically inspired solutions are greatly applicable for recovering coverage issues and efficient routing processes. In HEED, to find a node with lowest communication cost, large number of iterations is needed, thereby leading to larger time duration for finding such node, and this is considered as the prevalent drawback, resulting in significant power consumption.
In O-LEACH, the cluster head selection is based on randomness, resulting in easy cluster failure. Hence, to overcome these issues, formulation of a novel clustering strategy, which increases the network lifetime and decreases the power consumption, is essential.

\section{Proposed BIO-LEACH Algorithm}

\subsection{Methodology}

The proposed distributed clustering algorithm possesses three distinct features. First, the given clustering area will be divided in to different clusters and each cluster will be assigned with priority, which is not observed in O-LEACH. The cluster which is highly sensitive and which needs frequent data recording will be given highest priority. The clusters in which the priority is assigned, takes multiple sensing and communication even in one cycle. But, the clusters where priority is not assigned, only one sensing and data transmission will be allowed for one cycle. Second, the clusters possessing priorities will have one cluster head $(\mathrm{CH})$ and two supporting cluster head $(\mathrm{SCH})$, but the clusters that do not have priorities will have only one cluster head. Third, the clusters possessing priorities will be possessing cluster nodes more than that of the clusters without priorities, so as to avoid cluster failure. In case of O-LEACH, it was proposed to connect two separate and longer distanced sensor networks using an optical and distributed fiber link, and every other clustering process is followed as per the traditional LEACH algorithm. The main difference between the 0LEACH and BIO-LEACH is that, clustering process in O-LEACH is as per the traditional LEACH algorithm, which results in reduced network lifetime, but, the proposed algorithm employs aforementioned novelties in clustering process, thereby results in improved network lifetime. Fig.2 shows the basic articulation of the BIO-LEACH algorithm.

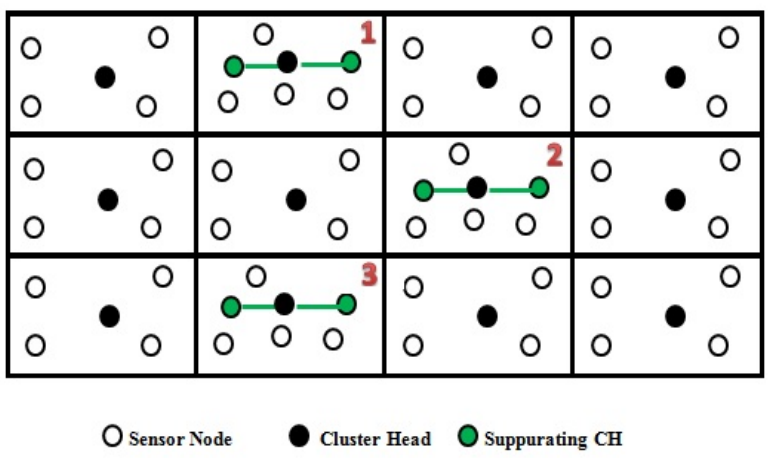

Figure 2: Basic articulation of BIO-LEACH algorithm.

The clustering field scenario considered for the proposed scheme is divided into 12 clusters of equal dimensions. Clusters 2 , 7 and 10 are only assigned with priorities, but the other clusters are considered as normal clusters. The clusters in which the priorities are assigned has one cluster head and two supporting cluster head $(\mathrm{SCH})$. Also, the sensor nodes are higher in number when compared with the ordinary clusters. The main reason we are assigning a greater number of sensor nodes is that, these priority clusters will do more sensing and data communication when compared to the ordinary clusters. The operational flowchart of the proposed algorithm is depicted in Fig.3. The three main phases in BIO-LEACH are cluster formation, Data transmission and $\mathrm{CH}-\mathrm{CH}$ communication. 


\subsection{Assignment of Priority and Synchronization}

In the proposed strategy, it is supposed for every node to possess a clock which varies from 0 to $\mathrm{T}$, and this could be expressed mathematically as,

$$
\phi_{i}(t)=\frac{t}{T} \phi_{i}(0) \bmod 1
$$

Where, I represent a particular node and $\phi_{i}(0)$ signify the offset period. Equation 1 clearly specifies that the state of a particular node at $t>0$, therefore the sensed information correlates with the firing time. The sensor node $i$ that hears the advertisement of its neighbouring nodes will update its clock based on the function given below,

$$
\phi_{j}\left(t^{+}\right)=\min \left\{f^{-1}\left(f\left(\phi_{j}(t)\right)+\varepsilon\right), 1\right\}
$$

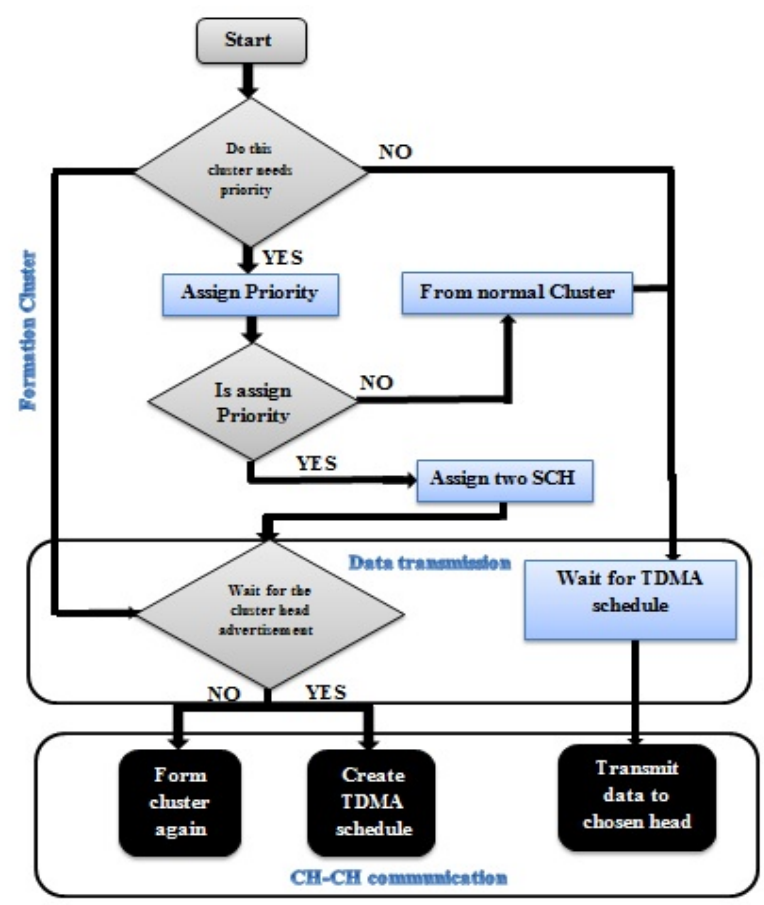

Figure 3: Operational flowchart of the Proposed Algorithm.

Where $\varepsilon>0$ represents the coupling strength and $f($.) represents the predestined sensor function such that $f(0)=0, f(1)=1$ and $f(x)>0 ; \forall x \in(0,1) \quad$ A typical example correlating the state $x_{j}$ and phase $\phi_{j}$ can be integrated as a function given below,

$$
\mathrm{x}_{\mathrm{j}}=f\left(\phi_{j}\right)=\frac{1}{b} \log \left(1+\left[e^{b}-1\right] \phi_{j}\right)
$$

A particular node $\mathrm{j}$ that hears the advertisement message shall be expressed and correlated mathematically as,

$$
\begin{gathered}
\phi_{j}\left(t^{+}\right)=\min \left\{a_{1} \phi_{j}(t)+a_{0}, 1\right\} \\
a_{1}=e^{b \varepsilon}
\end{gathered}
$$

$$
a_{0}=\frac{e^{b \varepsilon}-1}{e^{b}-1}
$$

Thus, the condition for an updated node $\mathrm{j}$ to get synchronized with the original node $i$ shall be integrated and expressed in mathematical form as below,

$$
\begin{aligned}
\phi_{j}\left(t^{+}\right) & =\min \left\{f^{-1}\left(f\left(\phi_{j}(t)\right)+\varepsilon\right), 1\right\} \\
\begin{array}{|lll|lll|}
\hline 0 & 0 & & 0 & 0 \\
0 & 0 & 0 & 0 & 0 \\
\hline
\end{array} & 0 \\
\text { Ordinary Cluster } & \text { Cluster with Priority }
\end{aligned}
$$

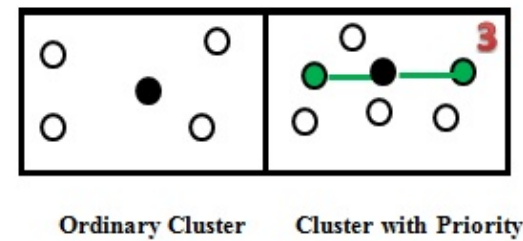

Figure 4: Cluster Formation Scenario.

Two different clusters are formed: one with priority and other normal clusters. Fig. 4 demonstrates how different types of clusters are formed in the proposed strategy. User can assign manually as per Equations (4)-(6), how many clusters needs priority and how many which do not need it, based on the requirements. Equations (1)-(7) describes the assignment of priority in the proposed algorithm, thereby forms an essential role when compared with $\mathrm{O}$ LEACH, in overcoming the drawbacks of O-LEACH.

\subsection{Data Transmission from $\mathrm{CH}$ to the $\mathrm{BS}$}

Fig.5 shows the data transmission path in the proposed approach. The cluster nodes will sense the data and keep it ready, once the TDMA time slot is assigned by the cluster head $(\mathrm{CH})$, these nodes forward them to the cluster head. The main difference between the existing algorithms and the proposed approach is that, the existing algorithms do not use relay nodes.

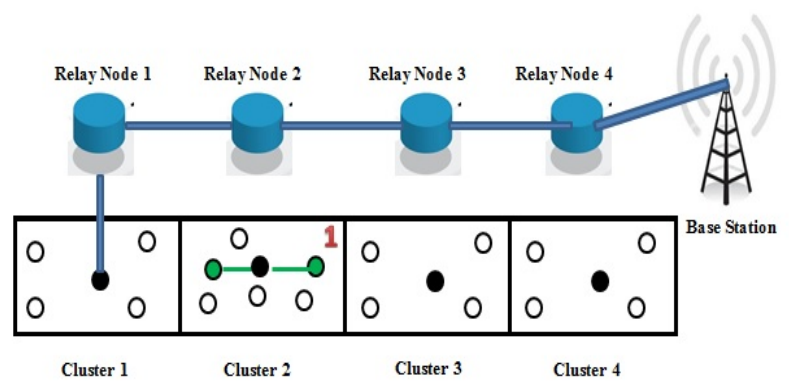

Figure 5:Data transmission path in the proposed approach.

Relay nodes are the nodes that are rich in resources, and in the proposed approach, these relay nodes are used only for communication between the $\mathrm{CH}$ and base station (BS). Relay nodes are employed between cluster head and the base station. These nodes carry the aggregated data from the cluster head to the base station. In most of the existing approaches, CHs sends the aggregated data to the base station, either directly or forwarding them through other cluster heads. This leads to sudden failure of cluster head. This shortcoming is avoided in the proposed approach, by the employment of relay nodes. Data transmission path is a multihop communication from $\mathrm{CH}$ to $\mathrm{BS}$. 


\section{Results and Discussions}

For simulation purpose, a square sensor field has been considered with $\mathrm{N}$ distinct sensor nodes with MxM dimension. The quantity of sensor nodes in the whole sensor field shall be expressed mathematically as,

$$
\mathrm{S}=\left\{\mathrm{s}_{1}, \mathrm{~s}_{2}, \ldots ., \mathrm{s}_{\mathrm{N}}\right\}
$$

The sensor nodes are assumed to be in immobile fashion, thereby each and every sensor nodes are pre-assigned with one unique identifier for distinguishing the data collected by the nodes in the sensor field, and can be expressed mathematically as,

$$
\mathrm{i} \in[1, \mathrm{~N}] \text { or } \quad 1 \leq i \leq N
$$

We also have assumed that every sensor nodes are heterogeneous and possess variable functionalities, and also the batteries of the sensor nodes are capable to recharge based on some mechanisms.

Table 1: The used Notations in BIO-LEACH Algorithm

\begin{tabular}{|l|l|l|}
\hline S.No & Notation & Description \\
\hline 1 & $C l_{-} m$ & Cluster Members \\
\hline 2 & $C H_{-} f(j)$ & Followers of $\mathrm{CH}$ i \\
\hline 3 & $C H_{-} c$ & CH Candidate \\
\hline 4 & $C H_{-} c(i)$ & Set of $\mathrm{CH}_{-} c$ received by sensor $\mathrm{i}$ \\
\hline 5 & $\varepsilon_{l}$ & Minimum Energy Level \\
\hline 6 & $\varepsilon_{t}$ & Quality Assessment Threshold \\
\hline 7 & $\varepsilon_{d}$ & Decay time \\
\hline 8 & $R_{c}$ & Communication Range \\
\hline 9 & $T_{-1}$ & Time of initialization step \\
\hline 10 & $T_{-2}$ & Time of CH Election step \\
\hline
\end{tabular}

The position $\left(\mathrm{x}_{\mathrm{i}}, \mathrm{y}_{\mathrm{i}}\right) \in \mathrm{R}$ of a sensor node $\mathrm{i}$ is signified and considered as $\theta^{i}$. Also, it is considered that, the sensor nodes do not know the exact position of other sensor nodes, but the cluster head and the sensor nodes are aware of the position of the base station. The BS is outside the sensors square, near the middle edge of the square, and has sufficient resources. Each sensor can receive a message from another, if it is in the communication range of the sender sensor. The communication distance corresponding to the sensing distance ds is expressed mathematically as,

$$
\mathrm{R}_{\mathrm{c}}=2 . \pi \times \mathrm{d}_{\mathrm{s}}
$$

For effective cluster formation in the proposed BIO-LEACH algorithm, the following parameters and notations are considered as represented in Table 1. Every simulation work has been done in NS2 for maximum of 100 rounds. Unlike O-LEACH and HEED approaches, the subsequent cluster heads are elected only when the threshold values of the prevailing cluster head become less than 1 . This concept is not available in most of the existing distributed clustering algorithms. Subsequent to initialization step, the election of cluster heads wills commences at particular time
T1. But, in case of next time duration T2, the sensor node that has the possibility to become a cluster head will send CHFOLLOW message. In circumstance when the sensor node acquires this candidature, it will estimate the hop length and hop count of each and every $\mathrm{CH}$. In case, when the cluster head do not receive the CHFOLLOW message, it will change its status from node $\mathrm{j}$ to normal node i. In case of steady state phase, the data gets aggregated by following TDMA slots. The communication between $\mathrm{CH}$ and $\mathrm{BS}$ is accompanied with the help of CDMA, but communication in between local clusters will be done by TDMA.

Fig. 6 shows the number of $\mathrm{CHs}$ and number of $\mathrm{CH}$ elections in BIO-LEACH during 100 runs. In the first 10 runs out of the 10 elected cluster head only 5 is finalized. In 20 runs, 6 get finalized out of 10 elections. In next stage, 5 get finalized out of 14 elections. Finally, in 100 runs, 4 get finalized out of 10 . At an average, $45.63 \%$ represents the average percentage of cluster head finalized out of those selected initially. This figure is a decent value, which specifies the novel strategies followed for cluster head selection in the proposed approach. The clusters where priority is not assigned, only one sensing and data transmission will be allowed for one cycle. The clusters possessing priorities will have one cluster head and two supporting cluster head, but the clusters that do not have priorities will have only one cluster head. This concept helps in proper rotation of $\mathrm{CHs}$ when compared with the existing approaches, thereby aids in better $\mathrm{CH}$ selection.

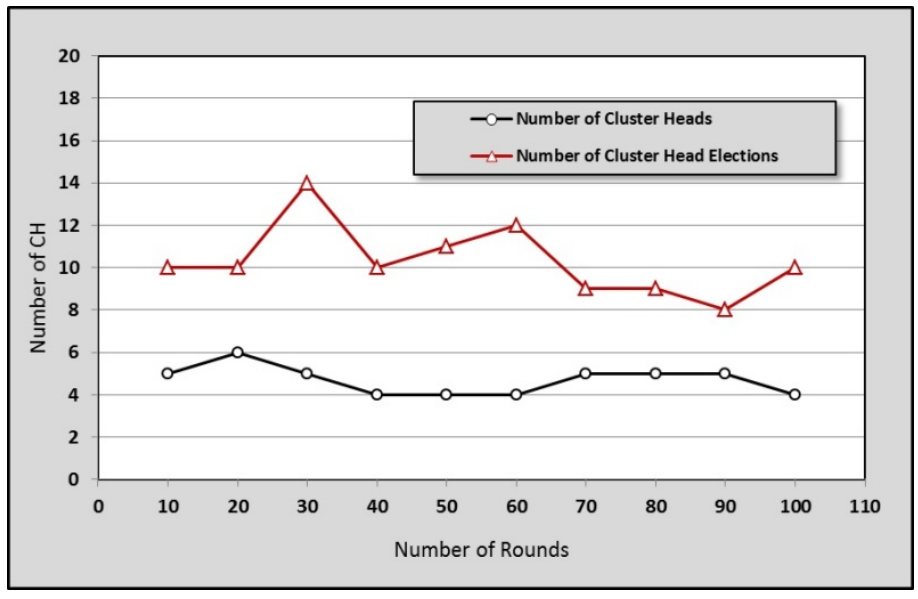

Figure 6: CH Election strategy outcomes.

Fig.7 specifies the network lifetime evaluation in BIO-LEACH. Network lifetime is nothing but the total span of period over which the sensor network will be alive. Also, in one angle, it could be specified as the time span for the first node to die, because the death of the first node implicitly indicates that there will be some loss in overall function of the sensor network. It also represents the case when, some network nodes die out of energy, and in this case the other network nodes being used to acquire the information from the dead node and transfer it to the base station. Here, initially for 1000 received packets, the number of nodes alive in O-LEACH, PSO and HEED are same as that of the proposed approach.

But, for 6000 received packets, the number of nodes alive in O-LEACH, PSO and HEED are 100, 400 and 450, but the proposed algorithm has a good number of alive nodes as 500 . 


\section{H.A.A AL-Asadi et al. / Advances in Science, Technology and Engineering Systems Journal Vol. 4, No. 5, $306-313$ (2019)}

Similarly, for 8000 received packets, the number of nodes alive in O-LEACH, PSO and HEED are 100, 200 and 350, but the proposed algorithm has a good number of alive nodes as 400 . Finally, for 10000 received packets, the number of nodes alive in O-LEACH, PSO and HEED are 100, 100 and 100, but the proposed algorithm has a good number of alive nodes as 200 . Thus, the loss of nodes is greatly prevented in the proposed approach. The average number of nodes alive in O-LEACH, PSO, HEED and BIO-LEACH are 2275, 3550, 4075 and 4325 . BIO-LEACH algorithm shows $47.39 \%$ improvement in number of nodes alive when compared to O-LEACH, $17.91 \%$ improvement in number of nodes alive when compared to PSO and 5.78\% improvement in number of nodes alive when compared to HEED, respectively. This is mainly achieved by the incorporation of relay nodes, priority and clustering concepts.

Here, the clusters possessing priorities will be possessing cluster nodes more than that of the clusters without priorities, so as to avoid cluster failure. In case of O-LEACH, it was proposed to connect two separate and longer distanced sensor networks using an optical and distributed fiber link, and every other clustering process is followed as per the traditional LEACH algorithm. Thus, by the concept of using priority, the proposed strategy attains betterment in network lifetime.

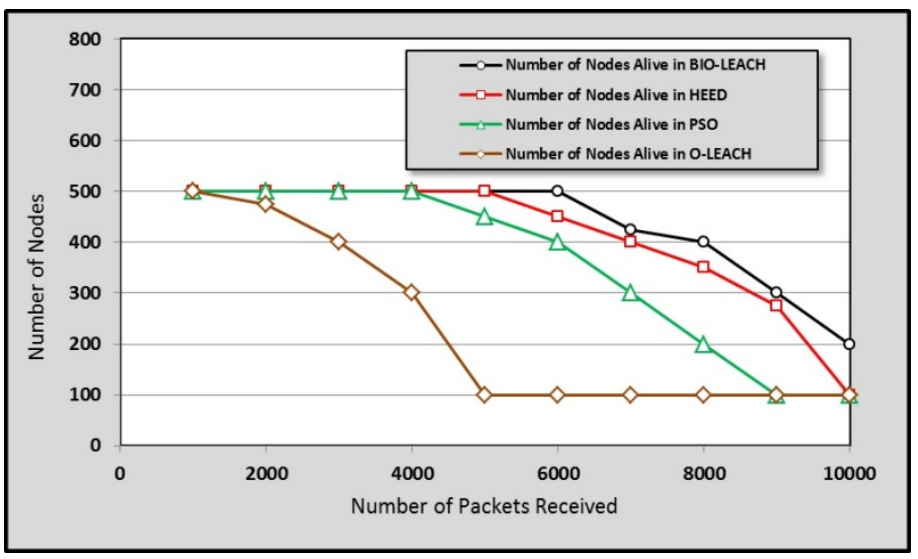

Figure 7: Network lifetime evaluation in BIO-LEACH.

Fig.8 describes the evaluation of amount of energy consumed in all the four approaches in terms or residual energies. Models proposed and implemented by Heinzelman normally attain better energy efficiency that implicitly signifies excellent power controlling and there is no any channel fading to happen. Initially for 1000 received packets, the residual energy in O-LEACH, PSO and HEED is 10 Joules, same as that of the proposed approach. But, for 6000 received packets, the residual energy in O-LEACH, PSO and HEED are 2 Joules, 4 Joules and 6 Joules, but the proposed algorithm has better residual energy as 5.5 Joules. Similarly, for 8000 received packets, the residual energy in OLEACH, PSO and HEED are 0.5 Joules, 2 Joules and 3 Joules, but the proposed algorithm has a better residual energy as 4 Joules. Finally, for 10000 received packets, the residual energy in OLEACH, PSO and HEED are 0.5 Joules, 1 Joule and 3 Joules, but the proposed algorithm has a good residual energy as 4 Joules. The average residual energy in O-LEACH, PSO, HEED and BIOLEACH are 38.50 Joules, 49 Joules, 60.50 Joules and 64.50 Joules. BIO-LEACH algorithm shows $40.31 \%$ betterment in residual energy when compared to O-LEACH, $23.87 \%$ improvement in residual energy when compared to PSO and 6.20\% improvement in residual energy when compared to HEED, respectively. For highly stable clusters, the residual graph should be linear with lesser irregularities, and from the graph it is evident that the proposed approach is highly stable.

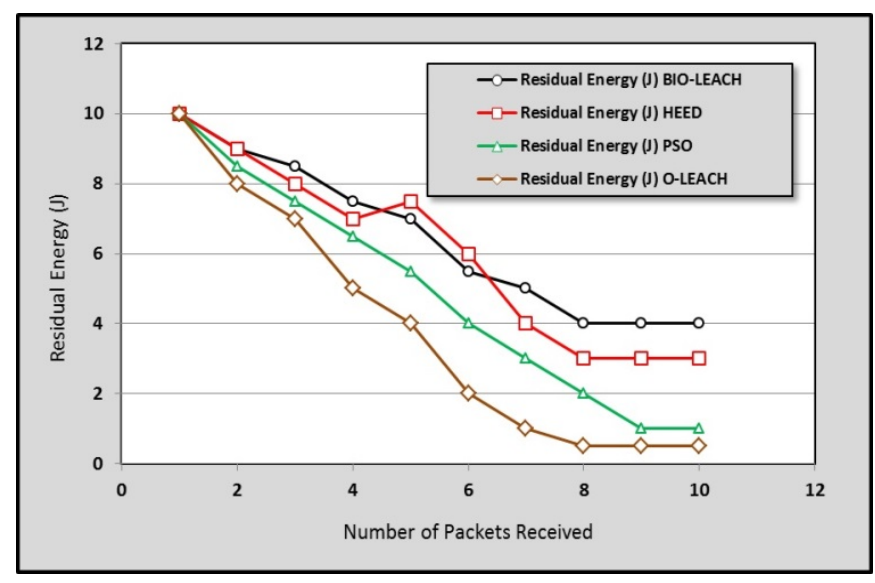

Figure 8: Evaluation of Amount of energy consumed.

Fig.9 describes the amount of packets received by the BS in all the four approaches. Congestion in wireless sensor network occurs because of loss or drop of packets, that mainly affects the network lifetime and throughput in these wireless sensor networks. Also, losses shall happen when the implemented network possess very poor security mechanisms. Initially for 10 rounds, the amount of packets received by the BS in O-LEACH, PSO and HEED is 10000 , same as that of the proposed approach. But, for 40 rounds, the amount of packets received by the BS in O-LEACH, PSO and HEED are 6600,6900 and 6400 , but the proposed algorithm has better amount of packets received by the BS as 8800 . Similarly, for 80 rounds, the amount of packets received by the BS in O-LEACH, PSO and HEED are 3900, 4200 and 3700, but the proposed algorithm has a better amount of packets received by the BS as 4 Joules. Relay nodes are employed between cluster head and the base station. These nodes carry the aggregated data from the cluster head to the base station. In most of the existing approaches, $\mathrm{CHs}$ sends the aggregated data to the base station, either directly or forwarding them through other cluster heads. This leads to sudden failure of cluster head. This shortcoming is avoided in the proposed approach, by the employment of relay nodes, thereby leading to number of successfully delivered packets. Also, this results in the decrease in communication delay, as seen in Fig.10.

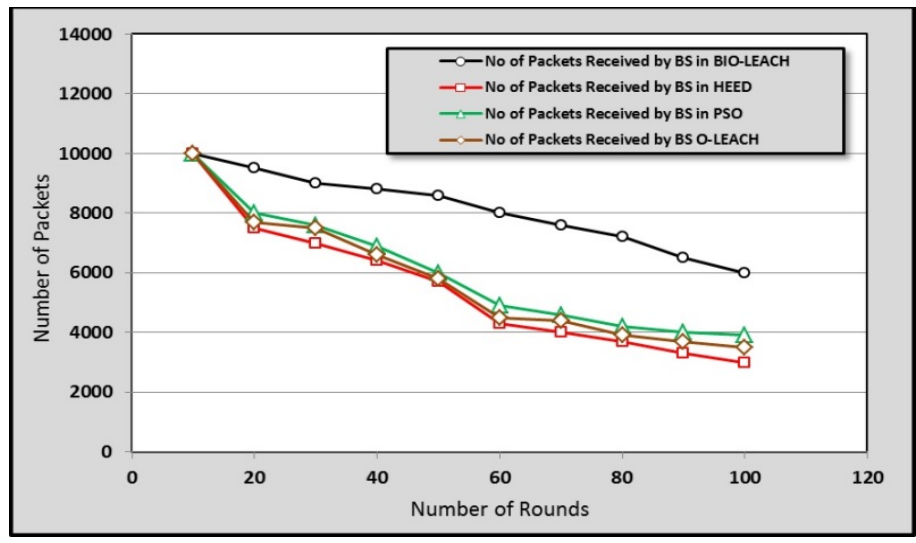

Figure 9: Amount of packets received by the BS in the Four Approaches. 


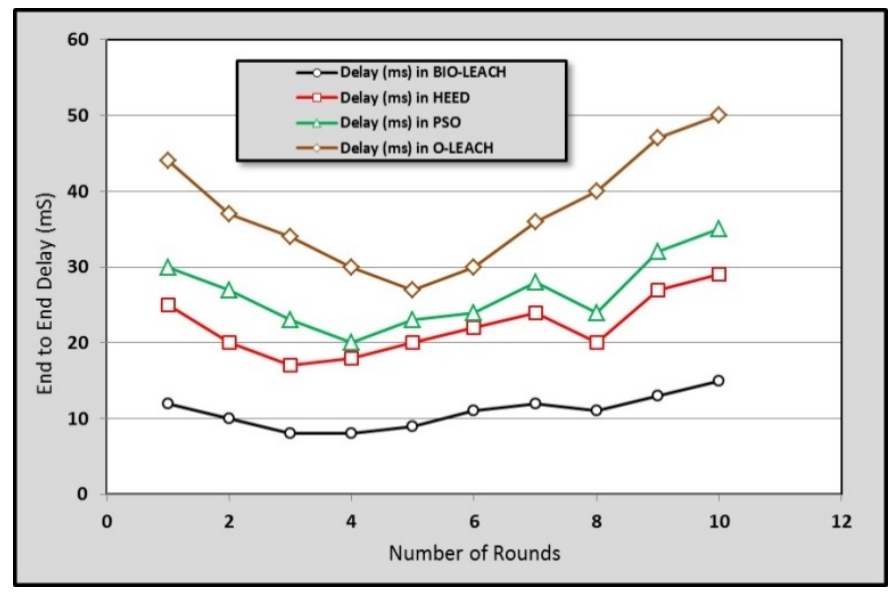

Figure 10: Average end-to-end delay during 100 runs for all the four Strategies.

Finally, for 100 rounds, the residual energy in O-LEACH, PSO and HEED are 3500, 3900 and 3000, but the proposed algorithm has a good amount of packets received by the BS as 6000 . The average amount of packets received by the BS in O-LEACH, PSO, HEED and BIO-LEACH are 57600, 60100, 54900 and 81200. BIO-LEACH algorithm shows $29.06 \%$ betterment in amount of packets received by the BS when compared to O-LEACH, 25.98\% improvement in amount of packets received by the BS when compared to PSO and $26.84 \%$ improvement in amount of packets received by the BS when compared to HEED, respectively. The slope of the curve is also linear with less irregularities, making it more stable.

Fig. 10 describes the average end-to-end delay during 100 runs for all the four Strategies. End-to-end delay or one-way delay signifies the overall time duration expended by the packets for reaching the base station from the cluster node or cluster head. It is a communal word in IP network monitoring, and varies from round trip time, where the path length in one way direction is only considered. Here, initially for 10 runs, the average end-to-end delay in O-LEACH, PSO and HEED is $44 \mathrm{~ms}, 30 \mathrm{~ms}$ and $25 \mathrm{~ms}$, but for the proposed approach the average end-to-end delay is $12 \mathrm{~ms}$. But, for 40 runs, the average end-to-end delay in O-LEACH, PSO and HEED are $30 \mathrm{~ms}, 20 \mathrm{~ms}$ and $18 \mathrm{~ms}$, but the proposed algorithm has reduced average end-to-end delay as $8.1 \mathrm{~ms}$. Similarly, for 80 runs, the average end-to-end delay in O-LEACH, PSO and HEED are $40 \mathrm{~ms}, 24 \mathrm{~ms}$ and $20 \mathrm{~ms}$, but the proposed algorithm has a low average end-to-end delay as $11 \mathrm{~ms}$. Finally, for 100 runs, the average end-to-end delay in O-LEACH, PSO and HEED are $50 \mathrm{~ms}$, $35 \mathrm{~ms}$ and $29 \mathrm{~ms}$, but the proposed algorithm has a reduced average end-to-end delay as $15 \mathrm{~ms}$. The average end-to-end delay in OLEACH, PSO, HEED and BIO-LEACH are $375 \mathrm{~ms}, 266 \mathrm{~ms}$, $222 \mathrm{~ms}$ and $109.1 \mathrm{~ms}$.

\section{Conclusion}

In this paper, a priority incorporated zone based distributed clustering algorithm, the Better Integrated and Optimized Low Energy Adaptive Clustering Hierarchy (BIO-LEACH) has been proposed for heterogeneous wireless sensor networks. This distributed clustering algorithm possesses three distinct features. First, the given clustering area will be divided in to different clusters and each cluster will be assigned with priority. The cluster which is highly sensitive and which needs frequent data recording will be given highest priority. The clusters in which the priority is assigned, takes multiple sensing and communication even in one cycle. But, the clusters where priority is not assigned, only one sensing and data transmission will be allowed for one cycle. Second, the clusters possessing priorities will have one cluster head $(\mathrm{CH})$ and two supporting cluster head $(\mathrm{SCH})$, but the clusters that do not have priorities will have only one cluster head. Third, the clusters possessing priorities will be possessing cluster nodes more than that of the clusters without priorities, so as to avoid cluster failure. From the simulation results, BIO-LEACH algorithm shows $47.39 \%$ improvement in number of nodes alive when compared to O-LEACH, $17.91 \%$ improvement in number of nodes alive when compared to PSO and 5.78\% improvement in number of nodes alive when compared to HEED, respectively. Also, the proposed strategy shows $40.31 \%$ betterment in residual energy when compared to O-LEACH, $23.87 \%$ improvement in residual energy when compared to PSO and $6.20 \%$ improvement in residual energy when compared to HEED, respectively. Moreover, the novel algorithm shows $29.06 \%$ betterment in amount of packets received by the BS when compared to OLEACH, $25.98 \%$ improvement in amount of packets received by the BS when compared to PSO and $26.84 \%$ improvement in amount of packets received by the BS when compared to HEED, respectively. Hence this proposed algorithm exhibits highly stable clusters.

\section{References}

[1] C. Han, Q. Lin, J. Guo, L. Sun and Z. Tao. "A Clustering Algorithm for Heterogeneous Wireless Sensor Networks Based on Solar Energy Supply", MDPI Electronics. 7(103), 1-20, 2018. https://doi.org/10.3390/electronics7070103

[2] R. Hou, L. He ; S. Hu ; J. LuoL., "Energy-Balanced Unequal Layering Clustering in Underwater Acoustic Sensor Networks", IEEE Access. (6). $39685-39691,2018$.

DOI: 10.1109/ACCESS.2018.2854276

[3] J. Corn, J. W. Bruce, "Clustering algorithm for improved network lifetime of mobile wireless sensor networks", IEEE International Conference on Computing, Networking and Communications (ICNC), 26-29, 2017. DOI: 10.1109/ICCNC.2017.7876282

[4] J. Yu, Y. Chen, L. Ma, B. Huang, and X. Cheng. "On connected target kcoverage in heterogeneous wireless sensor networks. Sensors", 16(1), 104, 2016.

DOI: $10.3390 / \mathrm{s} 16010104$

[5] A. Sharma and J. Thakur. "An energy efficient network life time enhancement proposed clustering algorithm for wireless sensor networks", In International Conference of Parallel and Distributed Computing Systems (PDCS 2014), 111- 115. 2014. Available: http://hpc-ua.org/pdcs-14/files/proceedings/17.pdf

[6] A.Ari, B. Yenke, N. Labraoui, I. Damakoa, and A.Gueroui. "A power efficient cluster-based routing algorithm for wireless sensor networks: Honeybees Swarm Intelligence based approach", Journal of Network and Computer Applications, 69:77-97, 2016. DOI: $10.1016 /$ j.jnca.2016.04.020

[7] X. Cai, Y. Duan, Y. He, J. Yang, and C. Li. "Bee-Sensor-C: An energyefficient and scalable multipath routing protocol for wireless sensor networks", International Journal of Distributed Sensor Networks, 0-14, 2015.

https://doi.org/10.1155/2015/976127

[8] R. Elhabyan and M. Yagoub. "PSO-HC: Particle swarm optimization protocol for hierarchical clustering in wireless sensor networks", In Collaborative Computing: Networking, Applications and Work-sharing (CollaborateCom), IEEE International Conference on, 417-424, 2014. DOI:10.4108/icst.collaboratecom.2014.257336

[9] R. de Friffein, R. Loomba, and B. Jennings. "Selecting energy efficient cluster-head trajectories for collaborative mobile sensing", In IEEE Global Communications Conference (GLOBECOM), 1-7. 2015.

DOI:10.1109/glocom.2014.7417727 
[10] Z. Al-Mekhlafi, Z. Hanapi, M. Othman, and Z. Zukarnain. "Travelling wave pulse coupled oscillator (TWPCO) using a self-organizing scheme for energy-efficient wireless sensor networks: PLoS One", 12(1), 01-27, 2017. https://doi.org/10.1371/journal.pone.0167423

[11] S. Shamshirband, N. Anuar, M. Kiah, V. Rohani, D. Petkovic, S. Misra, and A. Khan. "Co-FAIS: Cooperative fuzzy artificial immune system for detecting intrusion in wireless sensor networks", Journal of Network and Computer Applications, 42:102(117), 2014. DOI:10.1016/j.jnca.2014.03.012

[12] L. Yan., W. Pan., B. Luo., X. Li. and J. Liu., "Modified Energy-Efficient Protocol for Wireless Sensor Networks in the Presence of Distributed Optical Fiber Sensor Link,” IEEE Sensors Journal, Vol. 11, 1815-1819, 2011. DOI: 10.1109/JSEN.2010.2104317

[13] O. Younis, and S. Fahmy, "HEED: A Hybrid Energy-Efficient Distributed Clustering Approach for Ad Hoc Sensor Networks," IEEE Transactions on Mobile Computing, 2004.

DOI: $10.1109 /$ TMC.2004.41

[14] Y. Guo, C. Xie, and Z. Jing, 'Improvement on LEACH by combining adaptive cluster head election and two-hop transmission', Proceedings of the 9th International Conference on Machine Learning and Cybernetics, 11-14, 2010.

DOI: 10.1109/ICMLC.2010.5580988

[15] Z. Haitao, and B. Wenshao, 'A clustering routing protocol for energy balance of wireless sensor network based on simulated annealing and genetic algorithm', International Journal of Hybrid Information Technology, 7(2), 71-82, 2014

http://dx.doi.org/10.14257/ijhit.2014.7.2.08

[16] L. Han, "LEACH-HPR: An energy efficient routing algorithm for heterogeneous WSN", Proceedings of the IEEE International Conference on Intelligent Computing and Intelligent Systems, 507-511, 2010. DOI:10.1109/ICICISYS.2010.5658274

[17] J. Hill, R. Szewczyk, A. , S. Hollar, D. Culler, K. Pister, 'System architecture directions for networked sensors', Proceedings of the 9th International Conference on Architectural Support for Programming Languages and Operating Systems, 93-104, 2000.

DOI:10.1145/356989.356998 